\title{
Analysis and Performance Evaluation of Dynamic Frame Slotted-ALOHA in Wireless Machine-to-Machine Networks with Energy Harvesting
}

\author{
Shuang Wu, Yue Chen, Kok K Chai \\ Queen Mary University of London \\ London, U.K. \\ Email: s.wu@se10.qmul.ac.uk \\ \{yue.chen, michael.chai\}@qmul.ac.uk
}

\author{
F. Vazquez-Gallego, J. Alonso-Zarate \\ Centre Tecnològic de Telecomunicacions de Catalunya \\ Castelldefels, Barcelona, Spain \\ Email: \{francisco.vazquez, jesus.alonso\}@cttc.es
}

\begin{abstract}
One of the main challenges of Machine-to-Machine (M2M) communications is to extend the network lifetime by either using energy efficient communication protocols or by making use of energy harvesting techniques. In this paper, we consider a wireless M2M network composed of a large group of end-devices with energy harvesting capabilities that periodically transmit data to a gateway. We have analyzed and evaluated the probability of delivery, which measures the ability to transmit data to the gateway, using the Dynamic Frame Slotted-ALOHA (DFSA) protocol at the MAC layer. We have validated the correctness of the analytical model by means of computer-based simulations using MATLAB. Results show how the probability of delivery is influenced by the energy harvesting rate, the number of end-devices, the capacity of the energy storage devices (e.g., batteries), and the minimum energy that an end-device needs to transmit data. It has been possible to identify optimal configuration regions to extend the lifetime of M2M networks.

Index Terms-Machine-to-Machine; energy harvesting; Dynamic Frame Slotted-ALOHA; Markov chain model; probability of delivery.
\end{abstract}

\section{INTRODUCTION}

Machine-to-machine (M2M) networks enable the autonomous communication between devices without human intervention. M2M networks constitute a fundamental part of the Internet of Things (IoT), which envisage to provide benefits to individuals, society, and industries via a wide variety of smart applications. One of the main characteristics of wireless M2M networks is their dependency on batteries to power-up the end-devices. In many situations, the maintenance cost is high due to batteries replacement once the network has been deployed as the number of end-devices is large and they may be installed in awkward positions to handle. Therefore, one of the challenges of M2M networks is to extend the lifetime of end-devices. There are two main methods of extending the lifetime of end-devices: (i) by minimizing the energy consumption devoted to wireless communications, and (ii) by using energy harvesting techniques that potentially enable perpetual operation. Both approaches can be used in combination. In this paper, we focus on the Medium Access Control (MAC) layer, which manages the access of the enddevices to the shared wireless communication channel, and the integration of energy harvesting into its operation. From the MAC point of view, scheduled solutions such as Time Division Multiple Access (TDMA) could be optimal from the energy perspective, since collisions can be totally avoided, in contrast to random-access protocols where collisions and random backoff periods can drain the battery of the enddevices. Unfortunately, the high number of end-devices that operate in M2M networks makes it difficult to create and maintain a centralized schedule and, consequently, the design of energy-efficient random access protocols is needed.

The inclusion of energy harvesting in the operation of the MAC is a promising technique to extend the lifetime of M2M networks based on wireless communications. Through energy harvesting, end-devices can capture energy from the environment and avoid the need to replace or recharge batteries periodically [1] [2]. However, the inclusion of energy harvesting opens new challenges in the design of MAC protocols for M2M networks. For example, since the harvested energy is not guaranteed continuously, end-devices might not operate normally due to temporary energy shortages. These open challenges have motivated different research activities. Several research works use Markov chain models for wireless sensor networks (WSN) with energy harvesting, e.g., [3], [4]. These models are suitable for a single end-device, but do not consider the more realistic scenario with more than one end-device operating in the same network.

One of the first works related to the MAC layer with energy harvesting was presented in [5], which studies the data queue stability for Carrier Sense Multiple Access (CSMA). The work in [6] evaluates the throughput and delay performance of the Distributed Coordinated Function (DCF) of the IEEE 802.11 standard with energy harvesting. The work in [7] analyzes the throughput in wireless networks with energy harvesting 
using slotted-ALOHA. It presents a Markov chain model that represents the energy available in a device assuming that data packets are generated following a Poisson distribution and that energy arrives in fixed amounts at random time instants.

Unfortunately, the operation of ALOHA, CSMA, and DCF is not suitable for dense M2M networks. The access mechanisms based on random backoff periods and idle listening (clear channel assessment) lead to congestion as the number of contending end-devices increases. An alternative for such networks are the Frame Slotted-ALOHA (FSA) and Dynamic Frame Slotted-ALOHA (DFSA) protocols. The work in [8] considers FSA to coordinate the transmission of data to a gateway in M2M networks with energy harvesting. It proposes an algorithm to estimate the harvestable energy and to predict the optimum sleep period to ensure that the energy consumed in each end-device is less than the harvested energy.

The works in [9] [10] analyze DFSA and FSA in data collection networks with energy harvesting capabilities. They propose a Markov chain to analyze the energy stored in a device and compute the probability of delivery. Results show that the probability of delivery increases with the energy harvesting rate. However, they do not evaluate how the probability of delivery changes with the capacity of the energy storage device (e.g., battery) and the minimum energy (or threshold) that a device needs to transmit data. This is the main motivation for the work presented in this paper, which aims to fill this gap with the following contributions:

1) We use a Markov chain model to analyze DFSA in M2M networks with energy harvesting for data collection.

2) We optimize the joint operation of the MAC protocol and energy harvesting strategy to prolong the network lifetime.

3) We evaluate the probability of delivery over the energy harvesting rate, the number of end-devices, the capacity of the energy storage devices, and the energy threshold.

The remainder of this paper is organized as follows. In Section II, the system model is described. In Section III, we formulate the theoretical model to analyze the operation of DFSA with energy harvesting. Section IV is devoted to validate the model and discuss the performance evaluation. Finally, Section V concludes the paper.

\section{SySTEM MODEL}

We consider a single-hop wireless network formed by one coordinator and $n$ end-devices, each equipped with an energy harvesting unit (EHU) and an energy storage device (ESD), e.g., rechargeable battery or capacitor. The data communication flow and the MAC protocol operation are described in subsection II.A, then followed by the energy consumption, the energy storage, and the energy harvesting models.

\section{A. Data Model and MAC Protocol Operation}

Periodically, every $T_{R}$ seconds, the coordinator collects data from the end-devices by initiating Data Collection Rounds (DCR). The end-devices operate in power saving mode, and wake-up periodically to maintain synchronization. This operation is exemplified in Figure 1. The coordinator broadcasts

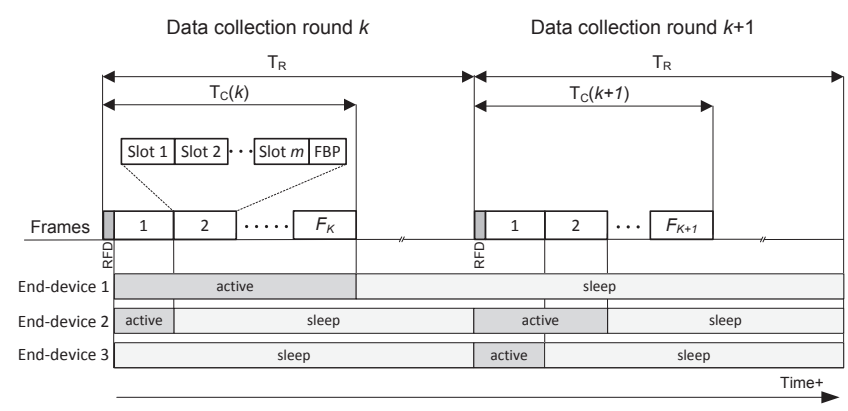

Figure 1: Illustration of data collection rounds considering $n=3$ end-devices.

a request for data (RFD) packet to initiate a DCR. Not all the end-devices will participate in each DCR. Only those end-devices whose energy level is above a predefined energy threshold will enter into active mode upon the reception of a RFD packet, and will try to transmit data to the coordinator. The rest will remain in sleep mode and wait for the next DCR.

The active end-devices will contend for the channel using the DFSA protocol to transmit exactly 1 data packet to the coordinator in a DCR. According to this protocol operation, time is divided into frames, which are further divided into $m$ slots where the end-devices transmit data. The active enddevices select at random one of the slots to transmit data in every frame. Each slot can be in three different states: (i) success, i.e., one data packet has been received and properly decoded by the coordinator; (ii) collision; no data packet can be decoded; or (iii) empty, the channel has been sensed idle.

We assume that the coordinator is able to estimate the number of end-devices that will contend in each frame [11] of a DCR. Then, according to DFSA, the coordinator adjusts the number of slots in every frame to be equal to the number of contenders. A feedback packet (FBP) is transmitted by the coordinator at the end of each frame to inform of: (i) the number of slots of the next frame, and (ii) the slots of the previous frame where a data packet has been successfully received. When an end-device has transmitted its data packet successfully, it enters into sleep mode until the next DCR starts. Otherwise, it reattempts transmission in the next frame as long as it has enough energy to do so. A DCR finishes when all the end-devices successfully transmit their data packet or go to sleep due to the lack of energy to remain active.

We assume that all packets are always transmitted without errors due to the wireless channel and there is no capture effect, i.e., a data packet cannot be decoded by the coordinator if it collides with other packets. We also assume that the time $T_{C}(k)$ of the contention process is shorter than the time $T_{R}$ between the beginning of two consecutive DCRs.

In the example of Figure 1, end-devices 1 and 2 have enough energy and they contend to transmit their data packet in the $k$-th DCR, while end-device 3 is in energy shortage at the beginning of the $k$-th DCR. In the $(k+1)$-th DCR, end-device 1 is in energy shortage, while end-devices 2 and 3 have enough energy to contend. 


\section{B. Energy Consumption and Energy Storage Models}

We normalize the energy consumed by the end-devices. Therefore, we say that an end-device consumes one energy unit per data packet transmitted in one frame (i.e., $E_{T X}=1$ ), regardless of the result of the transmission (i.e., success or collision), considering that this includes the energy used to transmit the data packet and receive the FBP. We consider that an end-device consumes 0 energy units in sleep mode.

The number of energy units stored in the ESD of an end-device is denoted by $E_{E S D} \in\{0,1,2 \ldots, N\}$, where $N$ indicates the normalized capacity of the ESD. $E_{E S D}$ can be modeled with a random variable which depends on the energy harvesting process and the energy consumption of the enddevices. We consider that the energy consumption is only due to radio operations.

An end-device enters in active mode at the beginning of the $k$-th DCR if its energy level, denoted as $E_{E S D}(k)$, is above a certain energy threshold $\varepsilon_{t h}$. Thus, the probability that an end-device enters in active mode in steady-state conditions (or probability of activation), denoted by $p_{\text {active }}^{\infty}$, can be expressed as

$$
p_{\text {active }}^{\infty}=\lim _{k \rightarrow \infty} \operatorname{Pr}\left\{E_{E S D}(k)>\varepsilon_{t h}\right\} .
$$

\section{Energy Harvesting Model}

The EHU captures an amount of energy from the environment, denoted by $E_{H}(k)$, along the time $T_{R}$ of the $k$ th DCR. The harvested energy $E_{H}(k)$ can be modelled as a discrete random variable with a probability mass function $q_{j}=\operatorname{Pr}\left\{E_{H}(k)=j\right\}$ with $j \in\{0,1,2, \ldots\}$, which depends on the characteristics of the energy source. The energy harvesting rate, denoted by $\overline{E_{H}}$, is defined as the average energy harvested by an end-device in a DCR,

$$
\overline{E_{H}}=\mathbb{E}\left[E_{H}(k)\right] .
$$

We assume that the energy harvested during the contention process $T_{C}$ of a DCR is negligible. We consider that the energy harvested by an end-device during a DCR is independent over end-devices and DCRs.

\section{ENERGy MODEL OF AN END-DEVICE}

The objective of this section is to present the markov chain that models the evolution of the energy available in an ESD, formulate the probability of success in a frame and the steadystate probability distributions, and derive the probability of delivery in a DCR.

\section{A. Discrete Markov Chain Model}

We use a two dimensional Markov chain to model the energy available in the ESD of an end-device as shown in Figure 2. Each state of the chain is defined by $\{e(t), f(t)\}$, where $e(t) \in\{0, \ldots N\}$ is a stochastic process which represents the number of energy units stored in the ESD at time $t$, and $f(t) \in\{0, \ldots N\}$ is a stochastic process which represents the number of frame in which an end-device contends. An enddevice is in sleep mode when $f(t)=0$.

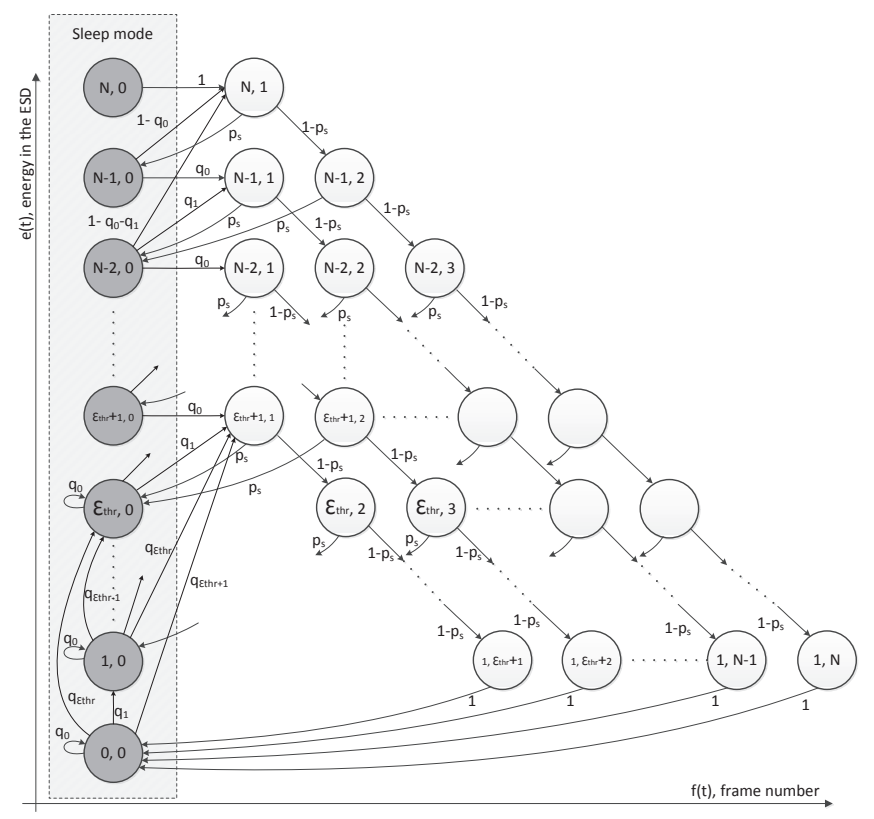

Figure 2: State transition diagram of the Markov chain that models the evolution of the energy available in an ESD. Some transitions have been intentionally omitted for ease of understanding.

The Markov chain is characterized by a transition matrix $P=\left[P_{i j}\right]$, where the one-step transition probabilities are defined as

$$
\begin{gathered}
P_{i j}=\operatorname{Pr}\left\{e(t+1)=e_{j}, f(t+1)=f_{j} \mid\right. \\
\left.e(t)=e_{i}, f(t)=f_{i}\right\} .
\end{gathered}
$$

The operations of an end-device across DCRs are as follows. When an end-device is not active in a DCR, it remains in sleep mode waiting for the next DCR. When a DCR starts, the amount of energy harvested during the previous $T_{R}$ is added. Then, if the energy available in the ESD is above the energy threshold $\varepsilon_{t h}$, the state of the end-device changes from sleep to active mode. See Figure 2 from $f(t)=0$ to $f(t+1)=1$. Otherwise, if the energy available in the end-device is still below or equal to the energy threshold when a DCR starts, the end-device remains in sleep mode.

The transition probability from the states in sleep mode is defined as

$P_{i j}=\left\{\begin{array}{l}q_{\varepsilon}, \quad \text { if }\left(e_{i} \leq e_{j}\right) \operatorname{and}\left(e_{j} \leq \varepsilon_{t h}\right) \operatorname{and}\left(f_{j}=0\right) \\ q_{\varepsilon}, \quad \text { if }\left(e_{i} \leq e_{j}\right) \operatorname{and}\left(\varepsilon_{t h}<e_{j}<N\right) \operatorname{and}\left(f_{j}=1\right) \\ 1-\sum_{k=0}^{N-1-e_{i}} q_{k}, \text { if }\left(e_{i}<e_{j}\right) \operatorname{and}\left(e_{j}=N\right) \operatorname{and}\left(f_{j}=1\right) \\ 0, \quad \text { otherwise, }\end{array}\right.$

where $q_{\varepsilon}$ is the probability that an end-device captures $\varepsilon=$ $\left(e_{j}-e_{i}\right)$ energy units. There are three different conditions in the transition from any state in sleep mode: (i) the end-device remains in sleep mode because it has not harvested energy, (ii) the end-device harvests $\varepsilon$ (with $e_{j}<N$ ) energy units, and (iii) the end-device harvests an amount of energy equal to or above the capacity of the ESD. 
Once an end-device becomes active at the beginning of a DCR, it will transmit its data packet in successive frames until it either succeeds in transmitting its data packet, enters in energy shortage, or both. The transition probability from any state in active mode is defined as

$$
P_{i j}=\left\{\begin{array}{l}
p_{s}, \quad \text { if }\left(e_{i}>1\right) \operatorname{and}\left(e_{j}=e_{i}-1\right) \operatorname{and}\left(f_{j}=0\right) \\
1-p_{s}, \quad \operatorname{if}\left(e_{i}>1\right) \operatorname{and}\left(e_{j}=e_{i}-1\right) \\
\quad \operatorname{and}\left(f_{j}=f_{i}+1\right) \\
1, \quad \operatorname{if}\left(e_{i}=1\right) \operatorname{and}\left(e_{j}=0\right) \operatorname{and}\left(f_{j}=0\right) \\
0, \quad \text { otherwise, }
\end{array}\right.
$$

where $p_{s}$ is the probability that an end-device succeeds in transmitting its data packet in a given frame, and $1-p_{s}$ is the probability that an end-device fails. There are three different conditions in the transition from any state in active mode: (i) if an end-device transmits its data packet successfully, it will change from state $\left(e_{i}, f_{i}\right)$ to $\left(e_{i}-1,0\right)$, (ii) if the enddevice does not succeed, it will change from state $\left(e_{i}, f_{i}\right)$ to $\left(e_{i}-1, f_{i}+1\right)$, and (iii) if the energy level of the end-device is 1 , it will change to $(0,0)$, no matter whether the transmission is successful or not.

\section{B. Probability of Success in a Frame}

The probability that an end-device succeeds in transmitting its data packet in a given frame, assuming that there are neither transmission errors nor capture effect, can be formulated as

$$
p_{s}=m\left(\frac{1}{m}\right)\left(1-\frac{1}{m}\right)^{n_{c}-1},
$$

where $m$ is the number of slots in the frame, and $n_{c}$ is the number of end-devices which contend in that frame. Since we consider that the number $m$ of slots in every frame is equal to the number $n_{c}$ of contending end-devices, then

$$
p_{s}=\left(\begin{array}{c}
n_{c} \\
k
\end{array}\right)\left(\frac{1}{m}\right)^{k}\left(1-\frac{1}{m}\right)^{n_{c}-k} \text { for } k=1,
$$

which is the binomial distribution with $k=1$. If $n_{c}$ is large, i.e., $n_{c}>20$, the expression of $p_{s}$ can be approximated by the Poisson distribution:

$$
p_{s} \simeq \frac{\lambda^{k} e^{-\lambda}}{k !}
$$

being $\lambda=n_{c} / m$ and $k=1$. Consequently, the probability that an end-device succeeds in a frame is constant, $p_{s} \simeq 1 / e$, for all the frames of the contention process of DFSA.

\section{Steady-State Probability Distributions}

The Markov chain model admits a unique steady-state probability distribution $\pi=\pi_{e, f}$ defined as

$$
\pi_{e, f}=\lim _{t \rightarrow \infty} \operatorname{Pr}\{e(t)=e, f(t)=f\},
$$

which satisfies that $\left(P^{\prime}-I\right) \pi^{\prime}=0$, where $P$ is the transition matrix and $I$ is the identity matrix. By calculating the eigenvector of $P^{\prime}$ that corresponds to an eigenvalue equal to 1 , we can solve the equation $\left(P^{\prime}-I\right) \pi^{\prime}=0$ and get $\pi$. Then, the probability of activation $p_{\text {active }}^{\infty}$ can be formulated as

$$
p_{\text {active }}^{\infty}=\pi_{\varepsilon_{t h}+1,1}^{B}+\pi_{\varepsilon_{t h}+2,1}^{B}+\cdots+\pi_{N, 1}^{B}=\sum_{e=\varepsilon_{t h}+1}^{N} \pi_{e, 1}^{B},
$$

where $\pi_{e, f}^{B}$ is the steady-state probability distribution conditioned on being active at the beginning of a DCR, which can be formulated as

$$
\pi^{B}=\pi^{S} P
$$

where $\pi^{s}$ is the steady-state probability distribution conditioned on being in sleep mode, which is calculated from the steady-state probability distribution as

$$
\pi_{e, f}^{S}= \begin{cases}\frac{\pi_{e, 0}}{\sum_{i=0}^{N} \pi_{i, 0}}, & \text { if }(f=0) \\ 0, & \text { if }(1 \leq f \leq N) .\end{cases}
$$

\section{Probability of Delivery}

The probability of delivery, denoted by $p_{\text {delivery }}^{\infty}$, is defined as the probability that an end-device becomes active and succeeds in transmitting its data packet in a DCR in steadystate conditions. Once the steady-state probability distribution conditioned on being active at the beginning of a DCR (9) is computed, we can formulate the expression to calculate $p_{\text {delivery }}^{\infty}$. Recall that an end-device that enters in active mode at the begining of a DCR starts from one of the states $(e, 1)$, with $e \in\left\{\varepsilon_{t h}+1, \ldots, N\right\}$, and then retransmits its data packet in the next frames as long as the available energy stored in ESD is enough. According to the steady-state probability distribution at the beginning of a DCR, the expression of the probability of delivery can be formulated as

$$
\begin{aligned}
p_{\text {delivery } y}^{\infty}= & \sum_{e=\varepsilon_{t h}+1}^{N} \pi_{e, 1}^{B} p_{s}+\sum_{e=\varepsilon_{t h}+2}^{N} \pi_{e, 1}^{B}\left(1-p_{s}\right) p_{s}+ \\
& \cdots+\pi_{N, 1}^{B}\left(1-p_{s}\right)^{N-1} p_{s} .
\end{aligned}
$$

which can be rewritten as

$$
\begin{gathered}
p_{\text {delivery }}^{\infty}=\sum_{f=1}^{\varepsilon_{t h r}} \sum_{e=\varepsilon_{t h r}+f}^{N} \pi_{e, 1}^{B}\left(1-p_{s}\right)^{f-1} p_{s}+ \\
\sum_{f=\varepsilon_{t h r}+1}^{N} \sum_{e=f}^{N} \pi_{e, 1}^{B}\left(1-p_{s}\right)^{f-1} p_{s} .
\end{gathered}
$$

\section{Model Validation And Performance EVALUATION}

In this section, we present and analyze the relationship between the probability of delivery and the following system parameters: (i) the energy harvesting rate; (ii) the total number of end-devices; (iii) the energy threshold to determine whether an end-device becomes active in a DCR; and (iv) the capacity of the ESD. In addition, we have compared the analytical results with the computer-based simulations using MATLAB to validate the analytical model proposed in section III. The simulation results of 500 samples have been averaged for every test case. Results show that the analytical results are tightly matched with the simulation results, thus validating the correctness of the analytical model. 


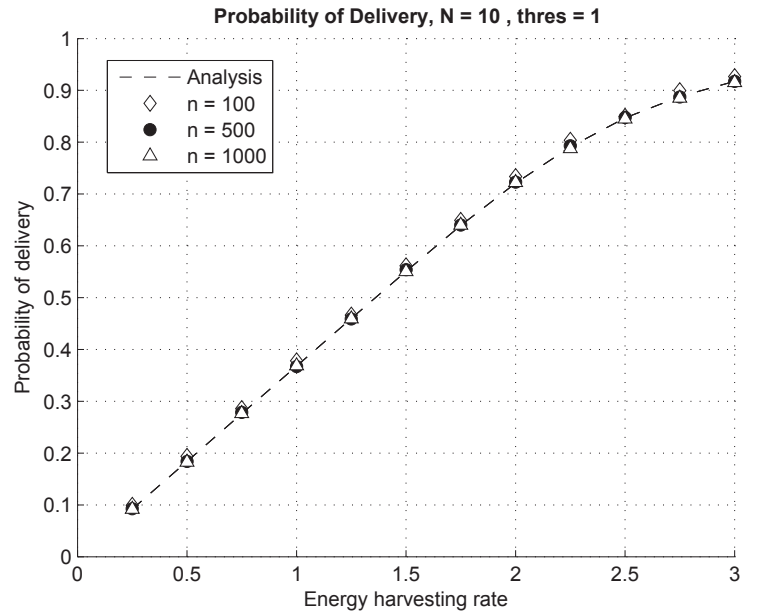

Figure 3: Probability of delivery over the energy harvesting rate.

We assume that the energy captured by an EHU follows a binominal distribution with probability mass function

$$
q_{j}=\left(\begin{array}{c}
N_{H} \\
j
\end{array}\right)\left(\frac{\bar{E}_{H}}{N_{H}}\right)^{j}\left(1-\frac{\bar{E}_{H}}{N_{H}}\right)^{N_{H}-j}
$$

for $j \in\left\{0,1,2, \ldots, N_{H}\right\}$, where $N_{H}=10$ is the maximum number of energy units that can be harvested, and $\bar{E}_{H} \in$ $\left[0,1,2, \ldots N_{H}\right]$ is the energy harvesting rate.

\section{A. Energy Harvesting Rate}

Figure 3 shows the probability of delivery as a function of the energy harvesting rate $\overline{E_{H}}$ (from 0.25 to 3 energy units). It has been evaluated by considering: $n \in$ $\{100,500,1000\}, \varepsilon_{t h}=1$, and $N=10$.

As it could be expected, the higher the energy harvesting rate, the greater the amount of energy that can be used to transmit data, and thus the greater the probability of delivery. The higher the number of energy units stored in the ESD between two consecutive DCRs, the greater the number of retransmissions is allowed.

It is worth noting that according to the analytical model presented in Section III, the probability of delivery is independent of the number of end-devices in the network. This can be corroborated with the simulation results shown in Figure 3. This is due to the fact that the average number of retransmissions of an end-device does not change with the number $n$ of end-devices. Note that the probability of success in a given frame is constant for all the end-devices thanks to the dynamic reconfiguration of the number of slots per frame of DFSA.

\section{B. Number of End-Devices}

Figure 4 shows the probability of delivery as a function of the number $n$ of end-devices (from 100 to 1000). It has been evaluated by considering: $\overline{E_{H}} \in\{0.5,1,1.5,2,2.5,3\}, \varepsilon_{t h}=$ 1 , and $N=10$.

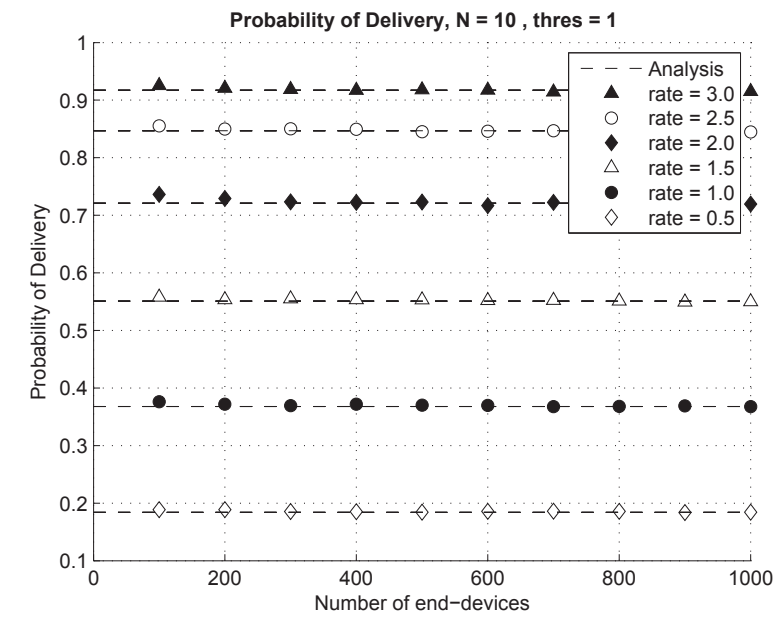

Figure 4: Probability of delivery over the number of enddevices.

As it can be observed in Figure 4, the result shows that the probability of delivery remains almost constant and independent of the number of end-devices. With DFSA, the number of slots in each frame is adjusted dynamically by the coordinator based to the number of contending end-devices in every frame, and thus the probability of collision among end-devices remains approximately constant.

In addition, it is possible to see that the higher the energy harvesting rate, the higher the probability of delivery. It can be observed that when the energy harvesting rate is lower than one energy unit, the probability of delivery is below 0.4 .

\section{Energy Threshold}

Figure 5 shows the probability of delivery as a function of the energy threshold $\varepsilon_{t h}$ (from 1 to 9 energy units), which is bound to the probability of activation in each DCR. It has been evaluated by considering: $n \in\{100,1000\}, \overline{E_{H}} \in$ $\{0.5,1,2,3,4,5\}$, and $N=10$.

The higher the energy harvesting rate, the more sensitive the probability of delivery is with regard of the selected threshold. On the contrary, the lower the energy harvesting rate, the less sensitive the probability of delivery becomes with respect to the threshold. The reason for this behavior lies in the fact that the higher the threshold, the more retransmissions are allowed per DCR since each end-device participates with more remaining energy in the ESD. However, when the energy threshold is too high, the average number of active enddevices per DCR is lower, and thus the probability of delivery decreases.

\section{Capacity of the Energy Storage Device}

Figure 6 shows the probability of delivery as a function of the capacity $N$ of the ESD (from 3 to 10 energy units). It has been evaluated by considering: $n \in\{100,500\}, \overline{E_{H}} \in$ $\{0.5,1,2,3\}$, and $\varepsilon_{t h}=1$. 


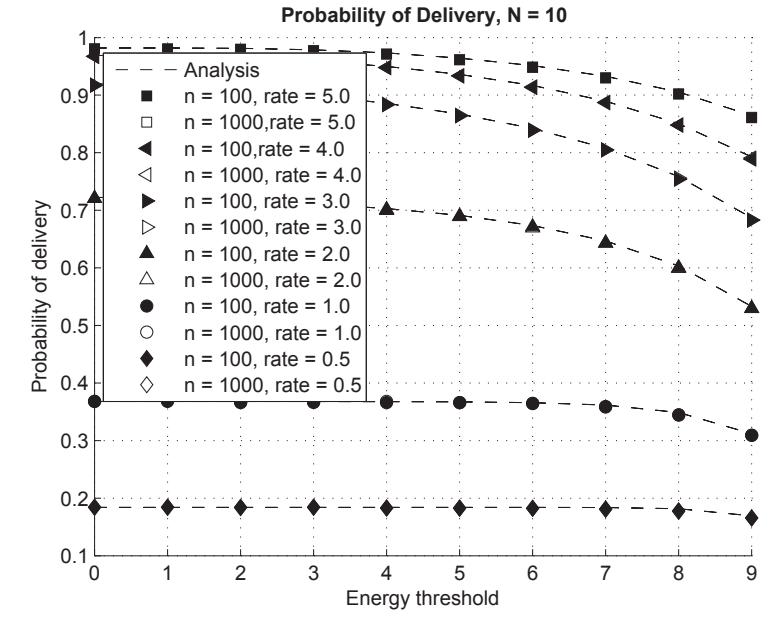

Figure 5: Probability of delivery over the energy threshold to enter in active mode at the beginning of each DCR.

Results show that the higher the capacity of the ESD, the higher the probability of delivery. For the higher energy harvesting rate (when $\varepsilon_{t h}$ is more than 2 energy units), the more sensitive the probability of delivery is with regard to the capacity of the ESD. On the contrary, the lower energy harvesting rate, the less sensitive the probability of delivery is with the increasing capacity of the ESD. When the capacity of the ESD is large, the amount of possible retransmissions per DCR is greater, and then the probability of transmitting a data packet successfully increases. In addition, the probability of delivery approximates to its maximum value when the capacity is above 6-7 energy units regardless of the energy harvesting rate. This is a very practical design guideline for the scenario considered in this paper.

\section{Conclusions}

In this paper, we have analyzed and evaluated the performance of M2M networks with energy harvesting capabilities using the Dynamic Frame Slotted-ALOHA (DFSA) protocol. In particular, we have considered the case when one coordinator requests periodic data from a number of enddevices with energy harvesters. Since energy is not always available at the end-devices, the number of contenting enddevices changes along time depending on the dynamics of the harvesting process. To understand the impact of such dynamic process in the performance of the network, we have considered the probability of delivery as a key performance indicator. Results show that DFSA efficiently optimizes the performance of the network as the number of contending end-devices changes. The impact of the size of the batteries and the energy harvesting rate has been shown, and it has been possible to identify optimal configuration regions to extend the lifetime of M2M networks. Future work aims at incorporating realistic channel models to the theoretical analysis and considering the possibility of transmitting more than one packet per DCR when fragmentation of data is required.

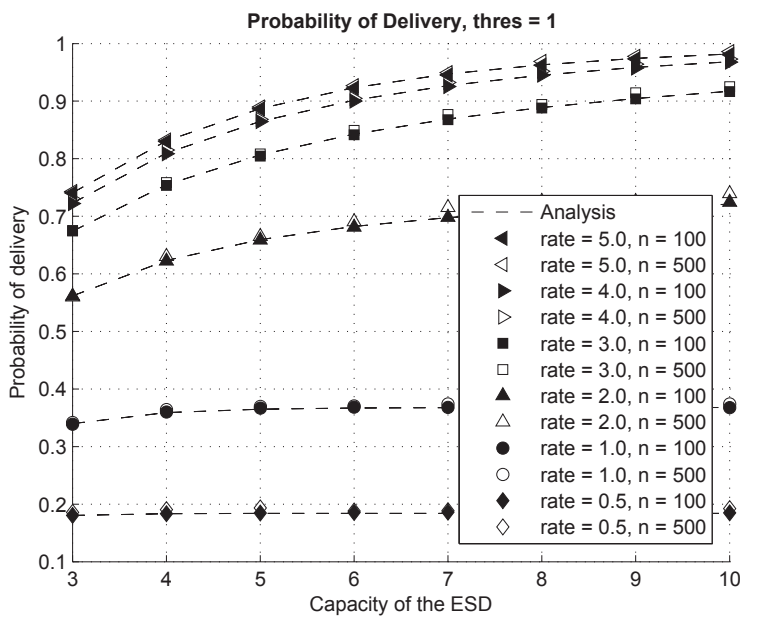

Figure 6: Probability of delivery over the capacity of the energy storage device.

\section{REFERENCES}

[1] S. Sudevalayam and P. Kulkarni, "Energy harvesting sensor nodes: Survey and implications," IEEE Communications Surveys Tutorials, vol. 13, no. 3, pp. 443-461, September 2011.

[2] M. Pinuela, P. Mitcheson, and S. Lucyszyn, "Ambient RF energy harvesting in urban and semi-urban environments," IEEE Transactions on Microwave Theory and Techniques, vol. 61, no. 7, pp. 2715-2726, July 2013.

[3] J. Lei, R. Yates, and L. Greenstein, "A generic model for optimizing single-hop transmission policy of replenishable sensors," IEEE Transactions on Wireless Communications, vol. 8, no. 2, pp. 547-551, February 2009.

[4] J. Ventura and K. Chowdhury, "Markov modeling of energy harvesting body sensor networks," in IEEE 22nd International Symposium on Personal Indoor and Mobile Radio Communications (PIMRC), September 2011, pp. 2168-2172.

[5] V. Sharma, U. Mukherji, and V. Joseph, "Efficient energy management policies for networks with energy harvesting sensor nodes," in Annual Allerton Conference on Communication, Control, and Computing, September 2008, pp. 375-383.

[6] G. Yang, G.-Y. Lin, and H.-Y. Wei, "Markov chain performance model for IEEE 802.11 devices with energy harvesting source," in IEEE Global Communications Conference (GLOBECOM), December 2012, pp. 5212 5217.

[7] M. Moradian and F. Ashtiani, "Throughput analysis of a slotted alohabased network with energy harvesting nodes," in IEEE International Symposium on Personal Indoor and Mobile Radio Communications (PIMRC), September 2012, pp. 351-356.

[8] O. Briante, A. M. Mandalari, A. Molinaro, G. Ruggeri, F. VazquezGallego, and J. Alonso-Zarate, "Duty-Cycle Optimization for Machineto-Machine Area Networks based on Frame Slotted-ALOHA with Energy Harvesting Capabilities," in European Wireless, May 2014.

[9] F. Iannello, O. Simeone, and U. Spagnolini, "Dynamic Framed-ALOHA for Energy-Constrained Wireless Sensor Networks with Energy Harvesting," in IEEE Global Telecommunications Conference (GLOBECOM), December 2010, pp. 1-6.

[10] F. Iannello, O. Simeone, P. Popovski, and U. Spagnolini, "Energy groupbased dynamic framed ALOHA for wireless networks with energy harvesting," in Annual Conference on Information Sciences and Systems (CISS), March 2012, pp. 1-6.

[11] B. Knerr, M. Holzer, C. Angerer, and M. Rupp, "Slot-wise maximum likelihood estimation of the tag population size in FSA protocols," IEEE Transactions on Communications, vol. 58, no. 2, pp. 578-585, February 2010 . 